\title{
Sclerostin is essential for alveolar bone loss in occlusal hypofunction
}

\author{
YANG XU $^{1 *}$, LUFEI WANG $^{1 *}$, YAO SUN $^{2}$, XIANGLONG HAN $^{2}$, TIAN GAO $^{3}, \mathrm{XIN} \mathrm{XU}^{1}$, \\ TIAN CHEN $^{1}$, XUEFENG ZHAO ${ }^{1}$, HUAN ZENG ${ }^{1}$, YANMIN WANG ${ }^{1}$ and DING BAI ${ }^{1}$
}

\begin{abstract}
${ }^{1}$ Department of Orthodontics, State Key Laboratory of Oral Diseases, West China Hospital of Stomatology, Sichuan University, Chengdu, Sichuan 610041; ${ }^{2}$ Department of Oral Implantology, Dental Transformation Medical Center, College of Stomatology;

${ }^{3}$ Department of Obstetrics and Gynecology, Transformation Medical Center, Tongji University, Shanghai 200011, P.R. China
\end{abstract}

Received November 22, 2014; Accepted December 23, 2015

DOI: $10.3892 / e t m .2016 .3124$

\begin{abstract}
Bone loss is caused by occlusal hypofunction and is a serious health concern. This is particularly true of tooth loss, which is common in the elderly. However, the cellular and molecular mechanisms underlying bone loss have yet to be fully elucidated. Sclerostin and $\mathrm{Wnt} / \beta$-catenin signaling have previously been reported to serve important roles in regulating bone remodeling. Therefore, the present study aimed to investigate the involvement of sclerostin and $\mathrm{Wnt} / \beta$-catenin signaling in occlusal hypofunction-induced alveolar bone remodeling. The unilateral maxillary molars of 14 male Sprague-Dawley rats were extracted in order to establish a model of occlusal hypofunction. For each rat, the non-extraction side was treated as the control group for comparisons with the extraction side. At 8 weeks after tooth extraction, the rats were sacrificed and alveolar bone specimens were harvested for X-ray radiography, micro-computed tomography (CT) and histological and immunohistochemical examinations. Bone loss and architecture deterioration were observed at the occlusal hypofunction side. The bone mineral density was markedly decreased and the ratio of bone volume to total volume was significantly decreased at the hypofunction side, as compared with the control side $(\mathrm{P}<0.001)$. In addition, the number of osteoclasts at the hypofunction side were significantly increased compared with that in the control side $(\mathrm{P}<0.001)$, as demonstrated using tartrate-resistant acid phosphatase staining. Furthermore, the protein expression levels of sclerostin and receptor activator
\end{abstract}

Correspondence to: Dr Yanmin Wang or Professor Ding Bai, Department of Orthodontics, State Key Laboratory of Oral Diseases, West China Hospital of Stomatology, Sichuan University, 14 Renmin South Road, 3rd Section, Chengdu, Sichuan 610041, P.R. China

E-mail: hxwym@scu.edu.cn

E-mail: baiding@scu.edu.cn

\section{"Contributed equally}

Key words: bone remodeling, sclerostin, mechanical unloading, occlusal hypofunction of nuclear factor $-\kappa \mathrm{B}$ ligand were increased, whereas those of $\beta$-catenin were decreased, at the hypofunction side when compared with the control side. In conclusion, the results of the present study suggested that occlusal hypofunction-induced bone loss may be associated with upregulated expression of sclerostin, which, in turn, may inhibit the activity of the Wnt/ $\beta$-catenin signaling pathway.

\section{Introduction}

Tooth loss is a common and serious health concern, particularly in the elderly. Although the incidence of tooth loss has declined in industrialized countries, the goal established by the World Health Organization of retaining 20 teeth at the age of 80 years has not been widely achieved $(1,2)$. Mechanical stress has a crucial role in bone remodeling $(3,4)$ and bone loss may occur as a result of reduced mechanical force, which is defined as disuse osteoporosis (5). Occlusal hypofunction due to tooth loss has a negative impact on jaw bone homeostasis (6). Tooth loss may lead to a reduced bite force in the antagonistic tooth and underlying alveolar bone during chewing. A lack of functional occlusion may induce active alveolar bone loss, including decreasing bone mass and volume $(7,8)$. In addition, in certain pathological conditions, such as estrogen deficiency, functional occlusion has been revealed to slow down the rate of bone loss (9), whereas occlusal hypofunction was reported to accelerate bone loss $(10,11)$. However, the cellular and molecular mechanisms underlying bone loss have yet to be fully elucidated.

The Wnt/ $\beta$-catenin signaling pathway has an essential role in bone remodeling by regulating osteoblast differentiation and function (12). Osteocytes, which are the most abundant cells in the bone, have a dominant role in sensing and transducing mechanical stress (13). Osteocytes express sclerostin, which is a SOST gene-encoded soluble protein that is thought to diffuse through the thick network of osteocyte canaliculi to reach the bone surface $(14,15)$. Previous studies have demonstrated that sclerostin, which typically antagonizes the Wnt/ $\beta$-catenin signaling pathway by binding to the LRP5/6 receptor to inhibit bone formation, is secreted less during mechanical stress, thus causing an increase in bone production in this context (16-18). In addition, sclerostin is able to promote osteoclastogenesis 


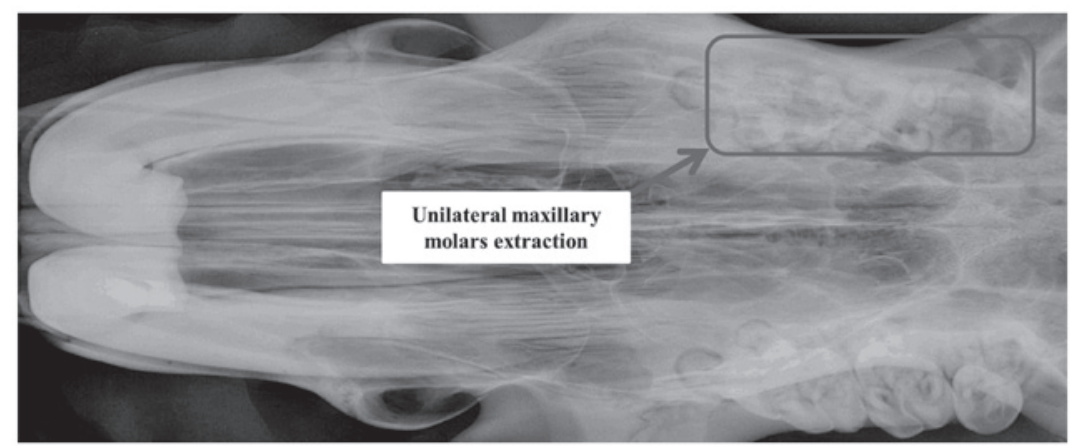

Figure 1. X-ray scan of the occlusal hypofunction model. The unilateral maxillary molars of 10-week-old male Sprague-Dawley rats were extracted to establish the model of occlusal hypofunction.

and osteoclast resorptive activity by a receptor activator of nuclear factor- $\kappa \mathrm{B}$ ligand (RANKL)-dependent pathway (19). In a model of tail suspension unloading-induced osteoporosis, decreased Wnt/ $\beta$-catenin signaling associated with upregulation of sclerostin was detected in the femur (20).

Alveolar bone is highly adaptable to the development of teeth and occlusal force, and is of unique character, as compared with the long bone of limbs $(6,7)$. Upon occlusion, the components of the tooth-periodontal ligament-alveolar bone complex behave in a synergistic manner (21). The reaction of alveolar bone to a mechanical stimulus may be affected by multiple components of the periodontium (8). Occlusal hypofunction has been reported to be a useful unloading model for jaw bone research, since it has been associated with tooth loss in patients requiring prosthodontic treatment $(10,11)$. However, to the best of our knowledge, the expression levels of sclerostin in the occlusal hypofunction environment have not been investigated to date. Therefore, the present study aimed to investigate the roles of sclerostin and Wnt/ $\beta$-catenin signaling in the regulation of alveolar bone loss induced by occlusal hypofunction.

\section{Materials and methods}

Establishment of a rat model of occlusal hypofunction. A total of 14 male Sprague-Dawley rats (age, 10 weeks) were used, sourced from Chengdu Da Shuo Biological Technology Co. Ltd. (Chengdu, China). All rats were fed a powder diet (Chengdu Da Shuo Biological Technology Co. Ltd.), had free access to drinking water and were maintained under a 12-12 h light-dark cycle at a constant temperature of $23^{\circ} \mathrm{C}$. The present study was approved by the Ethics Committee of the West China Hospital of Stomatology, Sichuan University (Chengdu, China). The maxillary molars of the rats were extracted on the left side, whilst the homolateral upper incisors were abraded, in order to induce the occlusal hypofunction environment (Fig. 1). For each rat, the non-extraction side was treated as the control group for comparisons with the extraction side. During the surgical procedure, anesthesia was administered intraperitoneally with $0.5 \%$ chloral hydrate (West China Second University Hospital, Chengdu, China). The rats were fed ad libitum throughout the experimental period. At week 8 after tooth extraction, all rats were sacrificed with an excess of chloral hydrate and the mandibles were collected and stored in a solution of $0.5 \%$ buffered formalin (Baoke Biotechnology, Inc., Chengdu, China).
Radiography and micro-computed tomography (CT) analyses. The whole mandibular alveolar bone was examined. The bone mineral density (BMD) of the alveolar bone was assessed by high resolution X-ray radiography using a Faxitron MX-20 Digital Radiography system (Faxitron Bioptics, LLC, Tucson, AZ, USA). The morphological characteristics of the alveolar bone were evaluated by micro-CT analysis. The specimens were placed in $10 \%$ buffered formalin and scanned using a desktop micro-CT system ( $\mu$ CT 35; Scanco Medical AG, Brüttisellen, Switzerland). The region of interest in the alveolar bone was selected and the bone volume (BV), total volume (TV) and the ratio of bone volume to total volume (BV/TV) were measured.

Histological evaluation and osteoclast activity. The alveolar bone samples were fixed overnight in $10 \%$ buffered formalin, decalcified in $0.5 \mathrm{~mol} / 1$ ethylene diaminetetraacetic acid ( $\mathrm{pH}$ 7.2) for 2 weeks at room temperature, and embedded in paraffin wax. Subsequently, 6- $\mu \mathrm{m}$ sections were prepared using an RM2235 microtome (Leica Microsystems GmbH, Wetzlar, Germany) and stained with hematoxylin and eosin (H\&E; Beijing Solarbio Science \& Technology Co., Ltd., Beijing, China).

Tartrate-resistant acid phosphatase (TRAP) staining was used to calculate the number of osteoclasts. Briefly, the sections were stained using a TRAP Staining kit (cat. no. 387A; Sigma-Aldrich, St. Louis, MO, USA) and the number of osteoclasts was calculated by two independent investigators using an Eclipse E100 microscope (Nikon Corporation, Toyko, Japan).

Immunohistochemistry. Demineralized, paraffin embedded sections of alveolar bone were prepared in order to detect the protein expression levels of sclerostin, $\beta$-catenin, osteoprotegrin (OPG) and RANKL by immunohistochemical staining. Briefly, the specimens were deparaffinized and treated with $3 \%$ hydrogen peroxide to inhibit endogenous peroxidase activity. Subsequently, the fixed sections were incubated with primary antibodies as follows: Polyclonal rabbit anti-sclerostin (dilution, 1:50; cat. no. ab63097; Abcam, Cambridge, UK), polyclonal rabbit anti-OPG (dilution, 1:200; cat. no. ab73400; Abcam) and monoclonal rabbit anti- $\beta$-catenin (dilution, 1:200; ab32572; Abcam) at $37^{\circ} \mathrm{C}$ for $2 \mathrm{~h}$, followed by incubation with horseradish peroxidase (HRP)-conjugated goat anti-rabbit immunoglobulin G (dilution, 1:200; sc-2004; Santa Cruz Biotechnology, Inc., Dallas, TX, USA) at $37^{\circ} \mathrm{C}$ for $30 \mathrm{~min}$. Alternatively, 

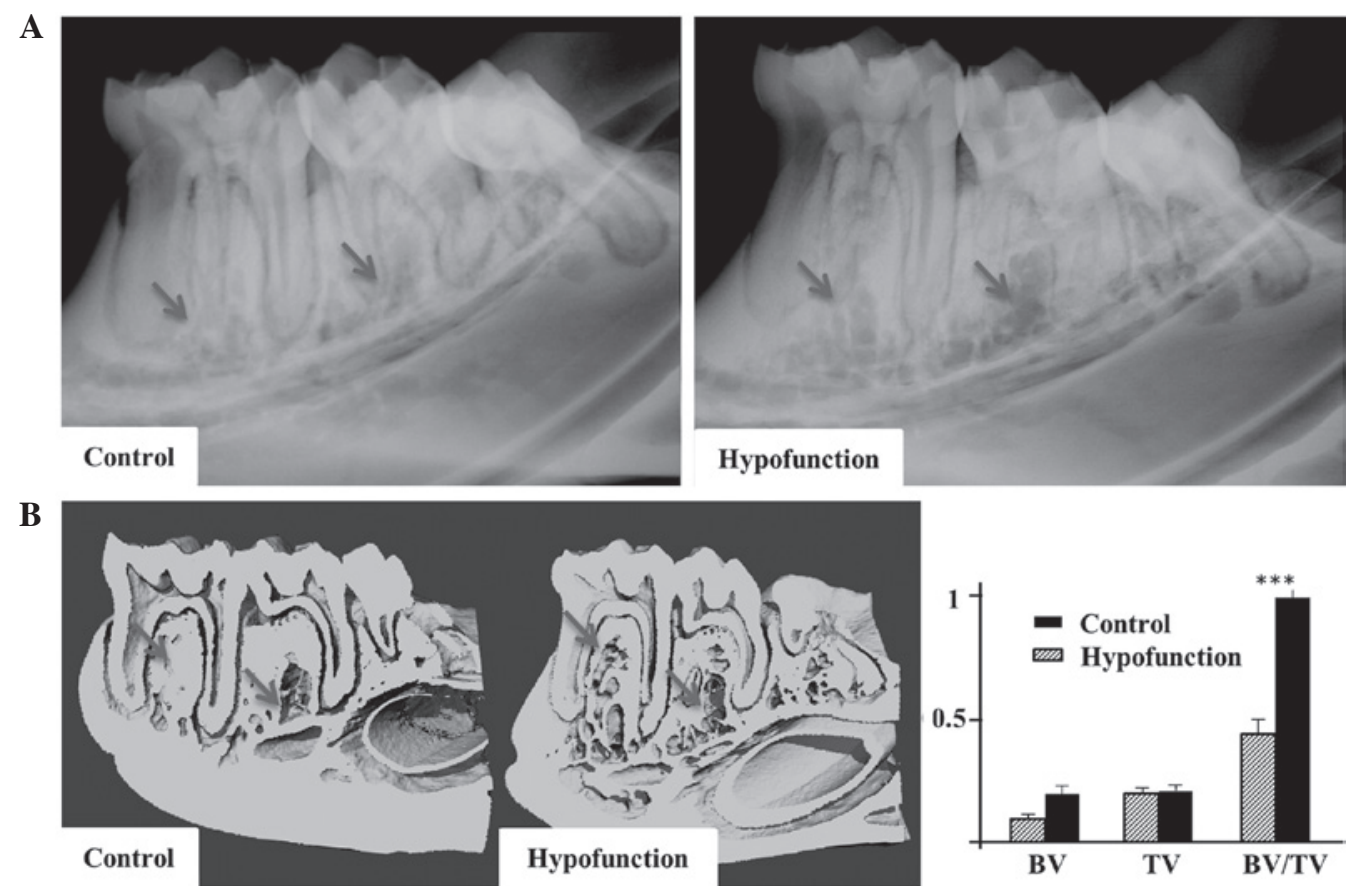

Figure 2. Histomorphometric analysis of alveolar bone. Bone alterations are indicated with arrows. (A) X-ray analysis detected decreased bone mineral density and active bone loss in the alveolar apical area at the hypofunction side. (B) Micro-computed tomography detected bone architecture deterioration and significantly reduced $\mathrm{BV}$ and $\mathrm{BV} / \mathrm{TV}$ values at the hypofunction side, as compared with the control side. ${ }^{* * * *} \mathrm{P}<0.001 \mathrm{vs}$. control side. $\mathrm{BV}$, bone volume; TV, total volume.

these were incubated with polyclonal goat anti-RANKL (dilution, 1:200; cat. no. sc-7628; Santa Cruz Biotechnology, Inc.), followed by HRP-conjugated rabbit anti-goat IgG within the Polink-2 Plus Polymer HRP Detection system (cat. no. PV9003; Zhongshan Bio-Tech, Co., Ltd, Beijing, China) for the corresponding duration and temperatures. Finally, the sections were stained using a 3,3'-diaminobenzidine tetrahydrochloride kit (OriGene Technologies, Inc.). Immunostained sections were scanned using a Nikon Eclipse 800 microscope (Nikon Corporation), and positive areas representing the degree of antigen expression were calculated using Image-Pro Plus version 6.0 image analysis software (Media Cybernetics, Inc., Rockville, MD, USA). Expression levels are presented as the mean optical density (MOD), which was calculated using the following equation: $\mathrm{MOD}=$ Integrated optical density / size of the study area.

Statistical analysis. All data are expressed as the mean \pm standard deviation. For quantitative outcomes, a paired t-test was used to compare the differences between each group. All statistical analyses were conducted using the SPSS software, version 17.0 (SPSS, Inc., Chicago, IL, USA). $\mathrm{P}<0.05$ was considered to indicate a statistically significant difference.

\section{Results}

Alveolar bone histomorphometric evaluation. A decreased BMD and bone destruction were observed in the alveolar apical area at the occlusal hypofunction side by X-ray analysis (Fig. 2A). In addition, high-resolution micro-CT images of the mandibles revealed decreased trabecular and cortical bone masses at the occlusal hypofunction side. Furthermore, development of a cavity and bone architecture deterioration were detected at the occlusal hypofunction side (Fig. 2B). According to quantitative analysis, the $\mathrm{BV}$ and $\mathrm{BV} / \mathrm{TV}$ values at the occlusal hypofunction side were significantly lower, as compared with those at the control side $(\mathrm{P}<0.001$; Fig. 2B). These results were indicative of severe bone loss at the occlusal hypofunction side.

Alveolar bone histological evaluation and osteoclast activity. Bone deterioration and resorption were observed at the occlusal hypofunction side by H\&E staining. In addition, a number of cavities were detected and the trabecular architecture appeared to be deteriorated (Fig. 3A). As demonstrated by TRAP staining, the number of osteoclasts was significantly higher at the occlusal hypofunction side, as compared with the control side $(\mathrm{P}<0.001$; Fig. $3 \mathrm{~B}$ and $\mathrm{C})$, thus indicating that active bone resorption was occurring.

Immunohistochemical results. As compared with the control side, the protein expression levels of $\beta$-catenin in the osteoblast cytoplasm were decreased, and those of sclerostin in the alveolar bone were increased, at the occlusal hypofunction side, as demonstrated by immunohistochemical staining (Fig. 4A and B). These results suggested that occlusal hypofunction suppresses the Wnt signaling pathway. Furthermore, positive RANKL immunoreactivity was detected in the osteoblasts and osteocytes, while positive OPG immunoreactivity was observed in the osteoblasts. At the hypofunction side, the protein expression levels of RANKL in osteocytes were significantly increased $(\mathrm{P}<0.001$; Fig. 5), as compared with the control side. By contrast, there was no significant difference in the protein expression levels of OPG between the sides ( $\mathrm{P}>0.05$; Fig. 5), although stronger immunoreactivity was detected at the hypofunction side. 
A

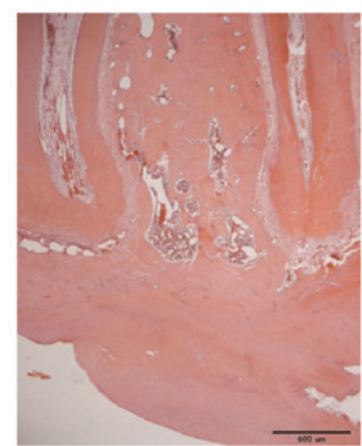

Control

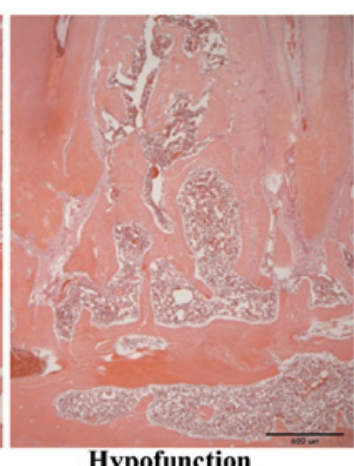

Hypofunction

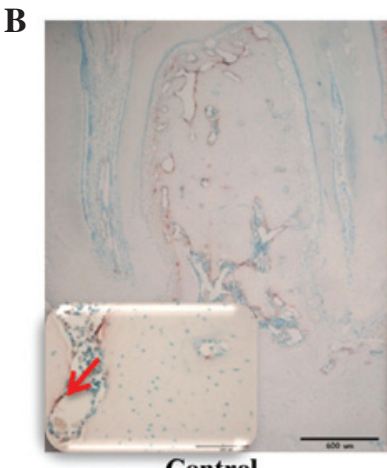

Control

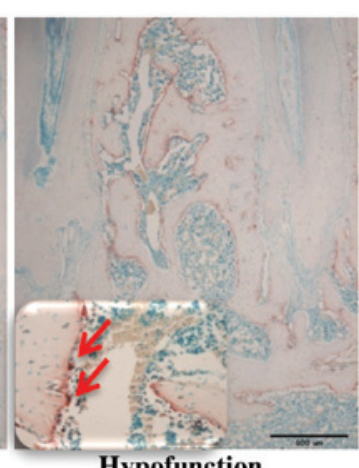

Hypofunction

C

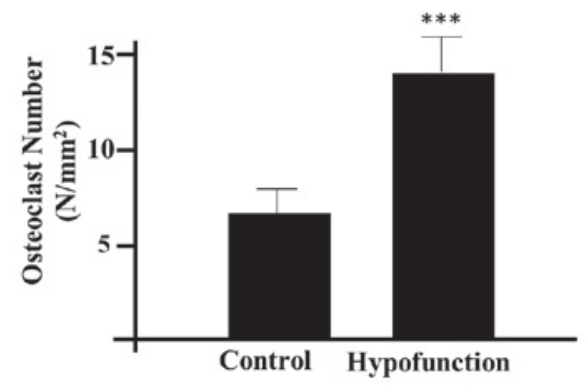

Figure 3. Histological evaluation and osteoclast activity. (A) Hematoxylin and eosin staining showed bone resorption occurred at the hypofunction side. (B and C) TRAP staining reflected a higher amount of osteoclasts (red arrows) at the hypofunction side $(\mathrm{P}<0.001)$. ${ }^{* * *} \mathrm{P}<0.001 . \mathrm{Magnification,} \mathrm{x} 100$.

A

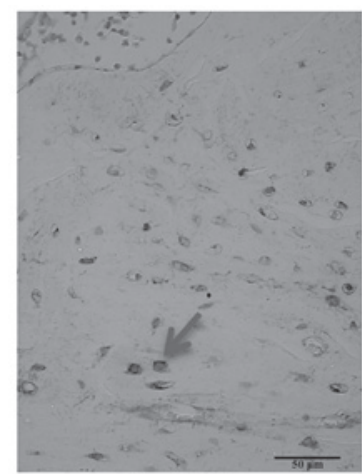

Control

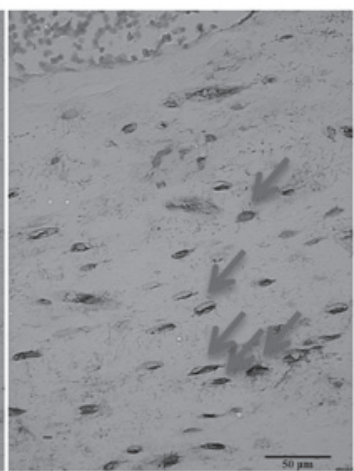

Hypofunction

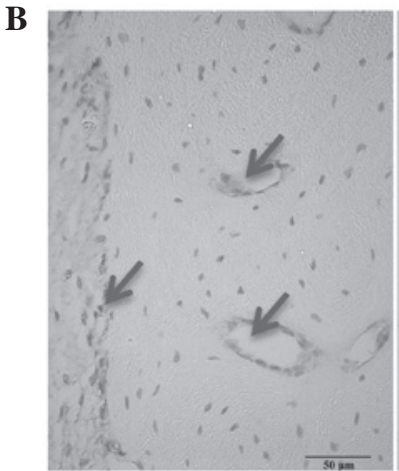

Control

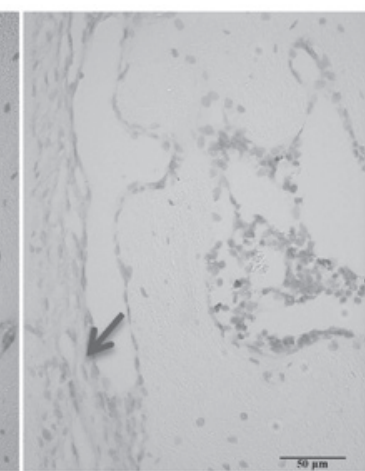

Hypofunction

Figure 4. Immunohistochemical staining of sclerostin and $\beta$-catenin. (A) Sclerostin and (B) $\beta$-catenin positive (+) cells in alveolar bone are indicated with arrows. The number of sclerostin+ cells were increased, and the number of $\beta$-catenin+ cells were decreased, at the hypofunction side, as compared with the control side. Magnification, x400.

\section{Discussion}

Tooth loss has been demonstrated to cause severe damage and may impair the oral health-associated quality of life of patients (22). Tooth removal may result in alveolar bone resorption and promote structural and compositional changes in the overlying soft tissue (23). In addition, tooth loss has been reported to diminish functional occlusion in the antagonistic tooth, which may lead to tooth extrusion and disuse osteoporosis of the alveolar bone $(7,8)$. Therefore, considering the potential complications during prosthetic rehabilitation, an evaluation of the magnitude of hard and soft tissue changes is essential prior to comprehensive prosthodontic treatment (24). In the case that the prosthetics rehabilitation is not applied immediately, these changes may deteriorate and have an unfavorable impact on the oral health. On the contrary, bone resorption can be reduced to some degree if the prosthesis is applied to balance the occlusal loading on the alveolar bone (25). In addition, the functional and cosmetic results of prosthodontic treatment depend on the quantity and quality of alveolar bone (26). Therefore, it is important to receive timely prosthetic restoration when tooth loss occurs.

The bone remodeling process consists of bone formation and bone resorption. Osteocytes serve a central role in the bone remodeling process by sensing and transmitting external mechanical loading information to effector cells, and function as an orchestrator in the regulation of osteoblast and osteoclast activities $(27,28)$. Numerous cell signalling molecules have been revealed to be modulated in osteocytes in response to mechanical stress, including adenosine triphosphate, nitric oxide, prostaglandin E2 and calcium channels (29). However, few of them have been demonstrated 

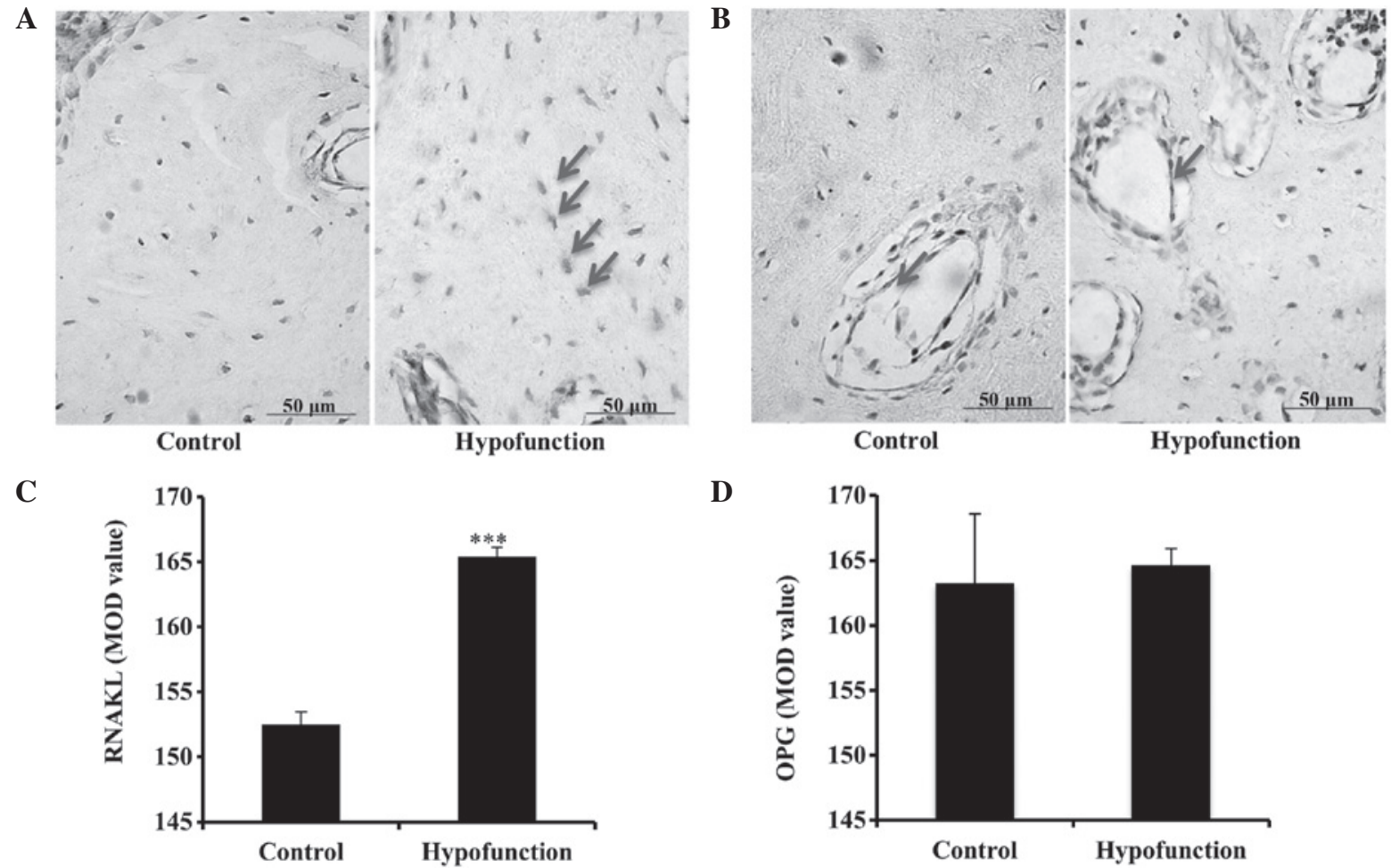

D

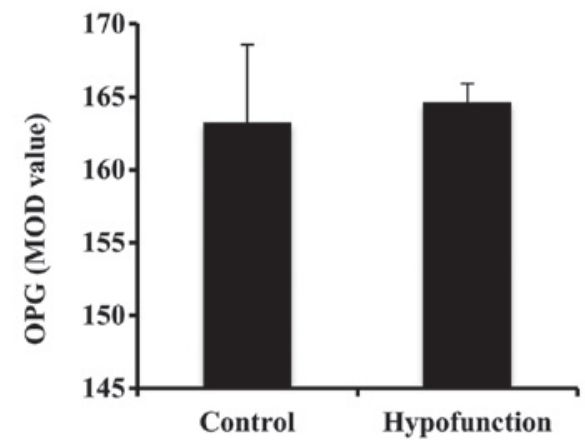

Figure 5. Immunohistochemical staining of RANKL and OPG. (A) RANKL and (B) OPG positive cells in alveolar bone are indicated with arrows. Quantitative immunohistochemical analysis of (C) RANKL and (D) OPG protein expression levels in the alveolar bone from the control and hypofunction sides. As compared with the control side, the protein expression levels of RANKL in osteocytes at the hypofunction side were significantly increased, whereas there were no significant difference in the protein expression levels of OPG between the sides. Data are presented as the mean \pm standard deviation of triplicate experiments. ${ }^{* * * *} \mathrm{P}<0.001$ vs. control side. Magnification, $\mathrm{x} 400$. RANKL, receptor activator of nuclear factor- $\mathrm{\kappa B}$ ligand; OPG, osteoprotegrin; MOD, mean optical density.

to be a prerequisite for loading adaptation (29). Previous studies demonstrated that loading reduces the secretion of sclerostin by osteocytes (30), which decreases the amount of sclerostin able to bind Lrp5/Lrp6 to antagonize the Wnt signaling pathway (31). Furthermore, downregulation of sclerostin in osteocytes has been shown to be an obligatory step in the osteogenic response to mechanical loading (32). In unloading models, such as in tail suspension, sclerostin expression levels increase, thereby inhibiting the Wnt signaling pathway, inducing apoptosis, suppressing osteoblast activity and decreasing the bone mass (20). Therefore, the current strategy of anti-sclerostin treatment for bone loss-associated diseases, as a potential therapeutic approach, has been investigated by numerous studies (33-36).

In the present study, evident bone loss and architecture deterioration were detected at the occlusal hypofunction side, as demonstrated using radiography, micro-CT and H\&E staining. A previous study reported that tail suspension unloading resulted in the upregulation of sclerostin (20), which was similarly observed in the present study in the occlusal hypofunction environment, since occlusal hypofunction is also an unloading environment. Upon extraction of the maxillary molars from the rats, the reduction in the bite force exposed the mandible to unloading. RANKL and OPG are recognized as positive and negative controllers of osteoclastogenesis, respectively (37). In the present study, the protein expression levels of RANKL were significantly increased in osteocytes at the hypofunction side. This is consistent with the study by Nakashima et al (38), which suggested that osteocytes are a crucial in vivo source of RANKL required for osteoclastogenesis. Conversely, the protein expression levels of OPG were not significantly different at the hypofunction side, as compared with the control side. It is hypothesized that the finding of stronger staining for OPG at the hypofunction side in the present study may have been the result of a negative feedback mechanism aimed to control the RANKL-promoted osteoclastogenesis process. These findings may suggest that bone resorption was facilitated. In addition, decreased $\beta$-catenin and increased sclerostin expression levels were observed in the occlusal hypofunction environment. Since sclerostin is an antagonist of the Wnt/ $\beta$-catenin signaling pathway $(39,40)$, the upregulation of sclerostin at the occlusal hypofunction side may have inhibited Wnt//-catenin signaling activity, thereby reducing bone formation, enhancing bone resorption and ultimately leading to bone loss.

The association of occlusal hypofunction with bone loss has been well-documented. The present study demonstrated that sclerostin-mediated $\mathrm{Wnt} / \beta$-catenin signaling inhibition may have a crucial role in this process, and supplied one potential molecular explanation for this phenomenon. The role of sclerostin-mediated Wnt/ $\beta$-catenin signaling in the regulation of unloading (tail suspension)-induced bone responses has previously been described in the long bone (20). However, occlusal unloading differs from tail suspension unloading. Typically, the mechanical stimulus is distributed among the teeth, periodontal ligament, and throughout the alveolar bone. Therefore, the loss of normal occlusal function leads not only to bone loss, but also to atrophic alterations in the periodontal ligament (41), which may also have an important role in alveolar bone loss and remodeling due to periodontal ligament fibroblasts responding to mechanical forces (42). 
Thus, the occlusal hypofunction model is required for jaw bone unloading experiments.

Future studies are required to investigate the roles of Wnt/ $\beta$-catenin, as well as numerous other signaling pathways, in the occlusal hypofunction status and their underlying mechanisms. Sclerostin is emerging as a promising therapeutic target in bone disease therapy (43). Various animal studies demonstrated that the sclerostin antibody was effective in preventing unloading-induced osteoporosis $(44,45)$. Thus, it can be hypothesized that anti-sclerostin treatment may be able to protect against occlusal hypofunction-induced alveolar bone loss, and its application in dental prosthetic rehabilitation may be promising.

In conclusion, the present study demonstrated that occlusal hypofunction-induced bone loss was associated with sclerostin and $\mathrm{Wnt} / \beta$-catenin signaling, in which upregulated sclerostin may antagonize the activity of the $\mathrm{Wnt} / \beta$-catenin signaling pathway, thereby inhibiting bone formation and accelerating bone resorption.

\section{Acknowledgements}

The present study was supported by the National Nature Science Foundation of China (grant nos. 81371171, 11172190, 81541112 and 81371172) and the Science and Technology Department, Sichuan, China (grant no. 2015FZ0074).

\section{References}

1. Recent advances in oral health. Report of a WHO Expert Committee. World Health Organ Tech Rep Ser 826: 1-37, 1992.

2. Müller F, Naharro M and Carlsson GE: What are the prevalence and incidence of tooth loss in the adult and elderly population in Europe? Clin Oral Implants Res 18 (Suppl 3): S2-S14, 2007.

3. Harada S and Rodan G: Control of osteoblast function and regulation of bone mass. Nature 423: 349-355, 2003.

4. Jacobs CR, Temiyasathit S and Castillo AB: Osteocyte mechanobiology and pericellular mechanics. Annu Rev Biomed Eng 12: 369-400, 2010.

5. Bikle DD and Halloran BP: The response of bone to unloading. J Bone Miner Metab 17: 233-244, 1999.

6. Shimomoto Y, Chung CJ, Iwasaki HY, Muramoto T and Soma K: Effects of occlusal stimuli on alveolar/jaw bone formation. J Dent Res 86: 47-51, 2007.

7. von Wowern N, Hjørting-Hansen E and Stoltze K: Changes in bone mass in rat mandibles after tooth extraction. Int $\mathbf{J}$ Oral Surg 8: 229-233, 1979.

8. Ejiri S, Toyooka E, Tanaka M, Anwar RB and Kohno S: Histological and histomorphometrical changes in rat alveolar bone following antagonistic tooth extraction and/or ovariectomy. Arch Oral Biol 51: 941-950, 2006.

9. Kuroda S, Mukohyama H, Kondo H, Aoki K, Ohya K, Ohyama T and Kasugai S: Bone mineral density of the mandible in ovariectomized rats: Analyses using dual energy X-ray absorptiometry and peripheral quantitative computed tomography. Oral Dis 9: 24-28, 2003

10. Elovic RP, Hipp JA and Hayes WC: Maxillary molar extraction decreases stiffness of the mandible in ovariectomized rats. J Dent Res 73: 1735-1741, 1994.

11. Elovic RP, Hipp JA and Hayes WC: Maxillary molar extraction causes increased bone loss in the mandible of ovariectomized rats. J Bone Miner Res 10: 1087-1093, 1995.

12. Glass DA II and Karsenty G: Molecular bases of the regulation of bone remodeling by the canonical Wnt signaling pathway. Curr Top Dev Biol 73: 43-84, 2006.

13. Tatsumi S, Ishii K, Amizuka N, Li M, Kobayashi T, Kohno K, Ito M, Takeshita S and Ikeda K: Targeted ablation of osteocytes induces osteoporosis with defective mechanotransduction. Cell Metab 5: 464-475, 2007.
14. Weidauer SE, Schmieder P, Beerbaum M,Schmitz W, OschkinatH and Mueller TD: NMR structure of the Wnt modulator protein sclerostin. Biochem Biophys Res Commun 380: 160-165, 2009.

15. Poole KE, van Bezooijen RL, Loveridge N, Hamersma H, Papapoulos SE, Löwik CW and Reeve J: Sclerostin is a delayed secreted product of osteocytes that inhibits bone formation. FASEB J 19: 1842-1844, 2005.

16. Bonewald LF and Johnson M: Osteocytes, mechanosensing and Wnt signaling. Bone 42: 606-615, 2008.

17. Galli C, Passeri G and Macaluso GM: Osteocytes and WNT: The mechanical control of bone formation. J Dent Res 89: 331-343, 2010.

18. Paszty C, Turner CH and Robinson MK: Sclerostin: A gem from the genome leads to bone-building antibodies. J Bone Miner Res 25: 1897-1904, 2010.

19. Wijenayaka AR, Kogawa M, Lim HP, Bonewald LF, Findlay DM and Atkins GJ: Sclerostin stimulates osteocyte support of osteoclast activity by a RANKL-dependent pathway. PLoS One 6: e25900, 2011.

20. Lin C, Jiang X, Dai Z, Guo X, Weng T, Wang J, Li Y, Feng G, Gao $\mathrm{X}$ and He L: Sclerostin mediates bone response to mechanical unloading through antagonizing Wnt/beta-catenin signaling. J Bone Miner Res 24: 1651-1661, 2009.

21. Naveh GR, Lev-Tov CN, Zaslansky P, Shahar R and Weiner S: Tooth-PDL-bone complex: Response to compressive loads encountered during mastication-a review. Arch Oral Biol 57: 1575-1584, 2012

22. Gerritsen AE, Allen PF, Witter DJ, Bronkhorst EM and Creugers NH: Tooth loss and oral health-related quality of life: A systematic review and meta-analysis. Health Qual Life Outcomes 8: 126, 2010.

23. Schropp L, Wenzel A, Kostopoulos L and Karring T: Bone healing and soft tissue contour changes following single-tooth extraction: A clinical and radiographic 12-month prospective study. Int J Periodontics Restorative Dent 23: 313-323, 2003.

24. Tan WL, Wong TL, Wong MC and Lang NP: A systematic review of post-extractional alveolar hard and soft tissue dimensional changes in humans. Clin Oral Implants Res 23 (Suppl 5): S1-S21, 2012.

25. Sennerby L, Carsson GE, Bergman B and Warfvinge J: Mandibular bone resorption in patients treated with tissue-integrated prostheses and in complete-denture wearers. Acta Odontol Scand 46: 135-140, 1988.

26. Bodic F, Hamel L, Lerouxel E, Baslé MF and Chappard D: Bone loss and teeth. Joint Bone Spine 72: 215-221, 2005.

27. Klein-Nulend J, Bakker AD, Bacabac RG, Vatsa A and Weinbaum S: Mechanosensation and transduction in osteocytes. Bone 54: 182-190, 2013.

28. Bonewald LF: The amazing osteocyte. J Bone Miner Res 26: 229-238, 2011.

29. Bonewald LF: Mechanosensation and transduction in osteocytes. Bonekey Osteovision 3: 7-15, 2006.

30. Robling AG, Niziolek PJ, Baldridge LA, Condon KW, Allen MR, Alam I, Mantila SM, Gluhak-Heinrich J, Bellido TM, Harris SE and Turner CH: Mechanical stimulation of bone in vivo reduces osteocyte expression of sost/sclerostin. J Biol Chem 283: 5866-5875, 2008.

31. Ellies DL, Viviano B, McCarthy J, Rey JP, Itasaki N, Saunders S and Krumlauf R: Bone density ligand, sclerostin, directly interacts with LRP5 but not LRP5G171V to modulate Wnt activity. J Bone Miner Res 21: 1738-1749, 2006.

32. Tu X, Rhee Y, Condon KW, Bivi N, Allen MR, Dwyer D, Stolina M, Turner CH, Robling AG, Plotkin LI and Bellido T: Sost downregulation and local Wnt signaling are required for the osteogenic response to mechanical loading. Bone 50: 209-217, 2012

33. Lewiecki EM: Role of sclerostin in bone and cartilage and its potential as a therapeutic target in bone diseases. Ther Adv Musculoskelet Dis 6: 48-57, 2014.

34. Virdi AS, Irish J, Sena K, Liu M, Ke HZ, McNulty MA and Sumner DR: Sclerostin antibody treatment improves implant fixation in a model of severe osteoporosis. J Bone Joint Surg Am 97: 133-40, 2015

35. Moe SM, Chen NX, Newman CL, Organ JM, Kneissel M, Kramer I, Gattone VH 2nd and Allen MR: Anti-sclerostin antibody treatment in a rat model of progressive renal osteodystrophy. J Bone Miner Res 30: 499-509, 2015.

36. Costa AG, Bilezikian JP and Lewiecki EM: The potential use of antisclerostin therapy in chronic kidney disease-mineral and bone disorder. Curr Opin Nephrol Hypertens 24: 324-329, 2015 . 
37. Tanaka Y, Nakayamada S and Okada Y: Osteoblasts and osteoclasts in bone remodeling and inflammation. Curr Drug Targets Inflamm Allergy 4: 325-328, 2005.

38. Nakashima T, Hayashi M, Fukunaga T, Kurata K, Oh-Hora M, Feng JQ, Bonewald LF, Kodama T, Wutz A, Wagner EF, et al: Evidence for osteocyte regulation of bone homeostasis through RANKL expression. Nat Med 17: 1231-1234, 2011.

39. Semënov M, Tamai K and He X: SOST is a ligand for LRP5/LRP6 and a Wnt signaling inhibitor. J Biol Chem 280: 26770-26775, 2005.

40. Li X, Zhang Y, Kang H, Liu W, Liu P, Zhang J, Harris SE and Wu D: Sclerostin binds to LRP5/6 and antagonizes canonical Wnt signaling. J Biol Chem 280: 19883-19887, 2005.

41. Muramoto T, Takano Y and Soma K: Time-related changes in periodontal mechanoreceptors in rat molars after the loss of occlusal stimuli. Arch Histol Cytol 63: 369-380, 2000.
42. Lekic P and McCulloch CA: Periodontal ligament cell population: The central role of fibroblasts in creating a unique tissue. Anat Rec 245: 327-341, 1996.

43. Ke HZ, Richards WG, Li X and Ominsky MS: Sclerostin and dickkopf-1 as therapeutic targets in bone diseases. Endocr Rev 33: 747-783, 2012.

44. Shahnazari M, Wronski T, Chu V, Williams A, Leeper A, Stolina M, Ke HZ and Halloran B: Early response of bone marrow osteoprogenitors to skeletal unloading and sclerostin antibody. Calcif Tissue Int 91: 50-58, 2012.

45. Spatz JM, Ellman R, Cloutier AM, Louis L, van Vliet M, Suva LJ, Dwyer D, Stolina M, Ke HZ and Bouxsein ML: Sclerostin antibody inhibits skeletal deterioration due to reduced mechanical loading. J Bone Miner Res 28: 865-874, 2013. 